\title{
Desigualdades de gênero e raciais no acesso e uso dos serviços de atenção primária à saúde no Brasil
}

\author{
Gender and racial inequalities in the access to and the use \\ of Brazilian health services
}

Barbara Cobo (https://orcid.org/0000-0003-4839-0776) ${ }^{1}$

Claudia Cruz (https://orcid.org/0000-0002-8788-3445) ${ }^{2}$

Paulo C. Dick (https://orcid.org/0000-0002-0744-7230) ${ }^{3}$

${ }^{1}$ Escola Nacional de Ciências Estatísticas. R. André Cavalcanti 106, Centro. 20231-050 Rio de Janeiro RJ Brasil. cobo. barbara@gmail.com

${ }^{2}$ Casa Fluminense. Rio de Janeiro RJ Brasil.

${ }^{3}$ Instituto Brasileiro de

Geografia e Estatística. Rio de Janeiro RJ Brasil.

\begin{abstract}
This paper aims to evaluate gender and racial inequalities in the access and use of health services in Brazil Based on the $2019 \mathrm{Na}$ tional Health Survey (PNS). Its main objective is to understand how white, black, or brown men and women seek medical care in Primary Health Care, the gateway to the Brazilian health system. Analyses from a gender perspective show that cultural and social patterns affect individual actions and choices and mainly access to and use of health services. The results also show that men and women reproduce the expected gender behavior, socially and culturally constructed, which impacts their self-assessment of health status, care, and their exposure to the risk of disease and death. The intersectional analysis reveals that racial inequalities are aligned with those observed between men and women, exponentiating vulnerabilities for self-identified black or brown people, reflecting the structural socioeconomic inequalities of Brazilian society. In this context, the universality and integrality recommended in the Unified Health System contribute as a public policy to the guarantee of rights, equalization of opportunities, and adequate access to equal care.
\end{abstract}

Key words Inequalities, Gender, Health services, Health policies
Resumo O presente artigo se propõe a avaliar, a partir das informações da Pesquisa Nacional de Saúde (PNS), 2019, as desigualdades de gênero e raciais no acesso e utilização dos serviços de saúde no Brasil. O objetivo principal é compreender como homens e mulheres, brancos, pretos ou pardos, buscam atendimento médico na Atenção Primária à Saúde, porta de entrada no sistema de saúde brasileiro. As análises sob uma perspectiva de gênero mostram que padrões culturais e sociais afetam ações e escolhas individuais e, em particular, o acesso e uso dos serviços de saúde. Os resultados também indicam que homens e mulheres reproduzem o comportamento de gênero esperado, social e culturalmente construído, que impacta sua autoavaliação de estado de saúde, cuidados e suas exposições ao risco de doença e morte. A análise interseccional revela que as desigualdades raciais se combinam às observadas entre homens e mulheres, exponenciando vulnerabilidades para pessoas de cor ou raça preta ou parda e refletindo as desigualdades socioeconômicas estruturantes da sociedade brasileira. Nesse contexto, a universalidade e a integralidade preconizadas no Sistema Único de Saúde contribuem enquanto política pública para a garantia de direitos, equalização de oportunidades e efetivação do acesso ao atendimento igualitário.

Palavras-chave Desigualdades, Gênero, Serviços de saúde, Políticas de saúde 


\section{Introdução}

No ano em que o Sistema Único de Saúde completou trinta anos de sua existência, toda a sua capacidade resolutiva, de cobertura, qualidade e efetividade foi posta à prova em função da pandemia da COVID-19. Para além das questões imediatas de saúde coletiva para prevenção, atendimentos e cuidados referentes à doença, os países precisaram lidar com os graves impactos sociais e econômicos em função das medidas de distanciamento social necessárias ao enfrentamento da pandemia, assim como a manutenção do acompanhamento de doenças crônicas e emergências médicas que, juntos, apresentavam risco potencial de colapsar as redes de atendimento à saúde.

No Brasil, o advento da pandemia em março de 2020 encontrou um sistema público de saúde universal em direitos no que concerne ao acesso a seus serviços, porém com graves distorções e gargalos de atendimento, em especial àqueles referentes à atenção primária de saúde, porta de entrada do Sistema Único de Saúde (SUS). Pontos nevrálgicos históricos do atendimento público hospitalar foram ainda mais expostos, como o Sistema de Regulação (SISREG) responsável pelas conhecidas filas de espera por consultas, exames e cirurgias; a lotação ou ausência de leitos hospitalares; a desigual distribuição de recursos e equipamentos e a clivagem público-privada no acesso à saúde.

É importante ressaltar que todo o cenário brevemente descrito se circunscreve em uma perspectiva mais abrangente de crise política e econômica que o país atravessa desde 2015, crise essa que afeta diferentemente mulheres, homens, pretos, brancos, pardos e indígenas nas mais diversas classes sociais. De fato, as desigualdades sociais constituem eixos estruturadores da sociedade brasileira, cujos resultados são refletidos nas diversas dimensões da qualidade de vida, restringindo acessos, oportunidades e a própria realização de direitos sociais legalmente constituídos. Durante a pandemia, muitas dessas desigualdades puderam ser evidenciadas, mostrando que grupos historicamente excluídos e em desvantagem social encontravam-se mais vulneráveis, não só aos riscos associados ao novo vírus, mas também ao desemprego, evasão escolar, pobreza e violência. Em particular, as desigualdades de gênero, principalmente quando analisadas em conjunto com a dimensão racial, ganharam contornos e nuances com poder de aprofundar iniquidades pré-existentes e revelar novas vulnerabilidades.
Ao trazer um panorama sobre o perfil epidemiológico e de atendimento da saúde em 2019, a divulgação da última edição da Pesquisa Nacional de Saúde (PNS) ${ }^{1}$ do Instituto Brasileiro de Geografia e Estatística (IBGE) se soma ao grande esforço de sistematização de informações fundamentais à compreensão da complexidade do período atual e contribui para o estudo da evolução no tempo dos indicadores de acesso à saúde pelos distintos grupos populacionais. Nesse sentido, o presente artigo buscou traçar uma análise do acesso e utilização dos serviços de atenção básica de saúde por meio dos dados coletados pela pesquisa, com enfoque nas desigualdades de gênero e raciais sempre que a representatividade da amostra permitiu a análise interseccional das informações. Os indicadores também foram comparados com a PNS $2013^{2}$ e com o suplemento da Pesquisa Nacional por Amostra de Domicílios $2008^{3}$, sempre que possível, de forma a trazer uma perspectiva temporal para a análise. Para tal, o artigo foi estruturado em quatro seções, além dessa Introdução: (1) A Atenção Básica de Saúde no Brasil e seus desafios; (2) Desigualdades de sexo e gênero no acesso e uso dos serviços de saúde; (3) Fatores determinantes para o acesso e uso dos serviços de saúde no Brasil; e (4) Conclusões.

$\mathrm{Na}$ seção (4), o cálculo dos indicadores e o ajuste do modelo de regressão logística foram efetuados utilizando o software R Project 4.0.0 $\mathrm{e}^{4}$ as funções do pacote survey ${ }^{5}$, considerando as informações do plano amostral complexo das pesquisas. A significância dos termos do modelo foi avaliada utilizando o teste de Wald ao nível 5\%, e a qualidade geral do ajuste foi verificada com o teste de Archer-Lemeshow ${ }^{6}$, também ao nível 5\%.

\section{A atenção básica de saúde no Brasil e seus desafios}

O direito universal à saúde, cuja garantia é prevista constitucionalmente como um dever do Estado, foi consubstanciado na criação e estruturação do Sistema Único de Saúde - SUS (Leis 8.080/1990 e 8.142/1990) e emergiu em um contexto de busca pela superação das desigualdades, segmentação e falta de acesso à saúde que eram críticos em décadas anteriores. Sob os princípios de universalidade, integralidade e descentralização, o SUS foi instituído com a premissa do atendimento independente da capacidade contributiva individual e, portanto, da sua inserção na lógica capitalista. Com orçamento próprio, que prevê fontes diversas de financiamento, o SUS vem, desde a sua fundação, sofrendo golpes à sua 
estrutura financeira através de medidas como a Desvinculação de Receitas da União (DRU) e desonerações da folha de pagamentos com impactos diretos nos recolhimentos destinados aos seu financiamento, cujo resultado tem sido a crescente precarização da qualidade e da oferta dos serviços públicos de saúde e um incentivo ao aprofundamento da clivagem público-privada no atendimento da saúde da população. $\mathrm{O}$ mais recente golpe ao financiamento do sistema público de saúde foi a Emenda Constitucional 95/2016 que congelou gastos sociais por 20 anos.

O SUS, organizado em função da complexidade dos seus atendimentos, tem como porta de entrada e eixo orientador do sistema a chamada Atenção Básica ou Atenção Primária, que se caracteriza por um conjunto de ações que englobam a promoção e a proteção da saúde, individual e coletiva, atuando no tratamento, na prevenção, no diagnóstico e na manutenção da saúde 7 . Neste eixo, estão as Unidades Básicas de Saúde (mais conhecidas como postos de saúde), as Clínicas de Saúde da Família e os Centros Municipais de Saúde. Os serviços constantes desse nível de atenção à saúde abrangem importantes programas, com destaque para o Saúde da Família, que atua através de uma equipe multiprofissional nas áreas de promoção de saúde de grupos específicos (Criança, Mulher, Idoso), controle e acompanhamento de doenças crônicas e saúde bucal.

É sobre o acesso e uso dos serviços de Atenção Primária que esse artigo delimita suas análises. Os últimos dados da PNS confirmaram a forte dependência da população aos serviços públicos de saúde, uma vez que $71,5 \%$ das pessoas declararam não ter acesso a planos privados de saúde médicos ou odontológicos em 2019, patamar similar ao encontrado pela pesquisa em 2013. Essa dependência era ligeiramente maior para homens $(72,6 \%$ contra $70,5 \%$ para mulheres), crianças e jovens $(74,5 \%)$, e substancialmente maior para pessoas pretas ou pardas (quase $80 \%$ contra $61,2 \%$ para brancos). Entre aqueles sem instrução ou fundamental incompleto, a dependência do SUS era de $84 \%$, uma diferença abissal para aqueles com superior completo, onde pouco mais de 30\% não tinham acesso planos de saúde privados. Desigualdades socioeconômicas que refletem desigualdades no acesso a serviços de saúde e que indicam a importância do sistema público como redutor de iniquidades.

Ademais, enquanto $76,5 \%$ das pessoas costumavam procurar o mesmo lugar, médico ou serviço de saúde quando necessitavam de aten- dimento, sete entre cada dez pessoas o procuravam no serviço público. A Estratégia de Saúde da Família, principal programa da Atenção Primária, apresentou uma expansão de sua cobertura, passando de 53,3\% de domicílios cadastrados no programa, em 2013, para 60,0\%, em 2019, com variações regionais importantes $(71,2 \%$ no Nordeste e $51,9 \%$ no Sudeste). Como cobertura de cadastro não caracteriza acesso e tampouco atendimento, houve queda no número de visitas domiciliares considerando os 12 meses anteriores à pesquisa. Em 2013, 47,2\% das unidades domiciliares cadastradas tiveram ao menos uma visita de algum membro da equipe de Saúde da Família, caindo para 38,4\%, em 2019, sendo que 23,8\% dos domicílios cadastrados não receberam sequer uma visita de agente comunitário ou membro da equipe de Saúde da Família no mesmo ano. Também houve queda na proporção de domicílios que receberam pelo menos uma visita de agente de combate a endemias (dengue, malária etc.) entre 2013 e 2019 (69,3\% e 64,6\% respectivamente).

De uma forma geral, os dados da PNS indicam uma maior procura por atendimentos de saúde entre 2013 e 2019, uma piora da percepção geral de saúde por parte da população e mais pessoas deixando de realizar atividades habituais por motivos de saúde. Todos esses indicadores com diferenciações importantes entre grupos populacionais e regionalmente, conforme veremos nas próximas seções.

\section{Desigualdades de gênero no acesso e uso dos serviços de saúde}

Análises preliminares dos dados da PNS 2019 corroboram resultados já conhecidos sobre diferenças de gênero no acesso e uso dos serviços de saúde, nos quais as mulheres tendem a procurar mais os serviços de saúde, seja para consultas preventivas de check up, seja pelas questões da saúde reprodutiva específicas, como pré-natal, preventivos etc. De acordo com a última edição da pesquisa, a proporção de mulheres que consultaram um médico nos 12 meses anteriores à realização da entrevista foi de $82,3 \%$, enquanto a porcentagem de homens foi de $69,4 \%$.

Para discutir possíveis fatores que determinam ou contribuem para esse comportamento diferenciado, importante pontuar que sexo e gênero não são termos substitutos ou intercambiáveis. Enquanto o primeiro remete ao sexo biológico masculino e feminino (ainda de forma dicotômica nas pesquisas amostrais domiciliares 
do IBGE), o segundo trata de uma construção social por meio de papéis e comportamentos esperados (ou não), aceitos (ou não) e valorizados (ou não) em função do primeiro. Nos estudos epidemiológicos, tanto o sexo quanto o gênero podem determinar condições de saúde e comportamentos diferenciados na utilização dos serviços, seja para cuidados, tratamento ou prevenção. No entanto, conforme aponta a epidemiologista $\mathrm{Krieger}^{8}$, é preciso entender que somos ao mesmo tempo "sexo" e "gênero": não vivemos como uma pessoa com "gênero" em um dia e um organismo com "sexo" no dia seguinte; nós somos ambos, simultaneamente, e para qualquer resultado de saúde dado, é uma questão empirica, não um princípio filosófico, se as diversas permutações de gênero e sexo importam - ou são irrelevantes. Gahagan et al. ${ }^{9}$, por sua vez, reconhecem que a integração sexo e gênero nas pesquisas fortalece a base geral de evidências de saúde e contribui para um planejamento mais adequado de ações e políticas públicas de saúde, para uma melhor adaptação dos cuidados e tratamentos e, dessa forma, para $o$ alcance da equidade no atendimento. Barata ${ }^{10}$ afirma que as desigualdades em saúde observadas entre homens e mulheres devem ser analisadas a partir dessa dupla determinação: as relações de gêenero e as peculiaridades do sexo biológico.

Empiricamente, os dados mostram que a mortalidade de homens tem sido superior à das mulheres em todas as faixas etárias do ciclo de vida, mas com destaque para as idades entre 15 e 29 anos, na qual a sobremortalidade masculina se justifica pela elevada incidência de mortes por causas externas (não-naturais). Comportamentos sociais associados ao gênero explicam em boa parte essa sobremortalidade, com homens culturalmente apresentando maior exposição a situações de risco no trabalho (ocupações com maior grau de insalubridade, esforço físico excessivo etc.) e na vida social (acidentes de carro e violências ou mesmo o uso excessivo de cigarros e bebidas alcoólicas). Mais uma vez, os resultados da PNS corroboraram esses comportamentos por meio das informações coletadas sobre alimentação adequada, uso de álcool, tabagismo e prática de atividade física (Tabela 1). As mulheres se alimentaram melhor e consumiram menos álcool e tabaco em 2019, não obstante o aumento observado do consumo em relação a 2013 (o consumo de álcool ao menos uma vez na semana passou de $12,9 \%$ para $17 \%)$. Por fim foram observados diferenciais de realização de atividades físicas entre homens e mulheres. Quando esta era realizada para fins de lazer ou trabalho, os homens eram mais ativos, porém, quando o nível de atividade física era realizado nos deslocamentos (compras, trabalho, levar crianças na escola, médico, buscar água etc.), as mulheres eram mais ativas que os homens.

Em contrapartida, considerando apenas informações em que a própria pessoa autoavaliou seu estado de saúde, mulheres tenderam a referenciar piores estados de saúde nos inquéritos populacionais. Na PNS 2019, enquanto 61,9\% das mulheres autoavaliaram seu estado de saúde como bom ou muito bom, esse percentual foi de $69,6 \%$ entre os homens. Pretos ou pardos referenciaram piores estados de saúde que brancos e as mulheres pretas ou pardas foram as que menos avaliaram seus estados de saúde como bom ou muito bom $(57,8 \%)$. Este diferencial por sexo e por cor ou raça se manteve ao longo da série histórica, inclusive com tendência de piora na autoavaliação de saúde para todos os grupos em relação a 2013 e 2008.

Para Barata ${ }^{10}$, embora algumas explicações para a maior morbidade referida entre mulheres estejam centradas em fatores biológicos, as questões de gênero precisam ser consideradas, uma vez que o papel universal e histórico conferido às mulheres como responsáveis pelos cuidados dos filhos e de outros membros da familia provavelmente determina a maior atenção para as questões de saúde e doença e, portanto, percepção mais aguçada para os próprios problemas. Outra questão apontada pela autora trata também da inserção laboral das mulheres, em geral, em ocupações mais precárias associadas a menores rendimentos, monotonicidade das tarefas, desgaste psicológico e emocional (inclusive com possibilidade de ocorrência de assédios morais e sexuais). Embora as mulheres apresentem carga horária de trabalho remunerado em média inferior à dos homens, ao se acrescentar as horas semanais dedicadas ao trabalho não remunerado (afazeres domésticos e cuidados), a carga conjunta de trabalho das mulheres (intensiva em trabalho manual) é superior à dos homens. De acordo com Cobo ${ }^{11}$ a soma de horas trabalhadas no mercado de trabalho, afazeres e cuidados das mulheres (carga horária de trabalho total) era superior à dos homens em 2,4 horas semanais. Essa dupla jornada pode estar por trás dos resultados da PNS que apontaram que $9,6 \%$ das mulheres deixaram de realizar atividades habituais por motivo de saúde $(6,3 \%$ dos homens), mas proporcionalmente foram os homens que mais deixaram de realizar tarefas habituais por motivos relacionados ao trabalho (16,1\% e $10,6 \%$ das mulheres). 
Tabela 1. Proporção de homens e mulheres de 18 anos ou mais de idade por dimensões de estilo de vida em função da exposição a fatores de risco à saúde - Brasil, 2019.

\begin{tabular}{lcc}
\hline \multicolumn{1}{c}{ Estilos de Vida } & $\begin{array}{c}\text { Homem } \\
\text { (\%) }\end{array}$ & $\begin{array}{c}\text { Mulher } \\
\text { (\%) }\end{array}$ \\
\hline Consumo recomendado de hortaliças e frutas & 10,2 & 15,4 \\
Consumo de alimentos minimamente processados & 22,9 & 24,4 \\
Consumo regular de refrigerantes & 11,6 & 7,2 \\
Consumo de bebida alcóolica ao menos 1x na semana & 37,1 & 17,0 \\
Consumo abusivo de álcool nos 30 dias anteriores & 26,0 & 9,2 \\
Direção após consumo de álcool nos últimos 12 meses & 20,5 & 7,8 \\
Usuários atuais de produtos derivados do tabaco & 16,2 & 9,8 \\
Nível recomendado de atividade física no lazer & 34,2 & 26,4 \\
Nível recomendado de atividade física no trabalho & 49,2 & 34,4 \\
Nível recomendado de atividade física no deslocamento & 31,2 & 32,2 \\
Nível recomendado de atividade física nas atividades domésticas & 9,1 & 21,8 \\
\hline
\end{tabular}

Nota: A PNS 2019 considerou como alimentação adequada a ingestão mínima de 5 hortaliças e 5 frutas (inclusive suco natural) por semana. $\mathrm{O}$ consumo regular de refrigerantes refere-se ao consumo referido por 5 dias ou mais por semana.

Fonte: IBGE, Pesquisa Nacional de Saúde 2019.

Segundo estudos desenvolvidos por Gomes et al. ${ }^{12}$ e Levorato et al..$^{13}$, os homens tendem a buscar atendimento médico somente quando há dores ou algum problema grave de saúde. Para além de questões culturais e de gênero, em que a construção cultural da masculinidade se sobrepõe às necessidades de cuidados e atendimento médico, homens alegam também que os horários de funcionamento das unidades públicas de saúde não condizem com suas realidades de trabalho, embora mulheres igualmente inseridas no mercado de trabalho acessem regularmente o sistema, inclusive de forma preventiva. Homens brancos com maior escolaridade e maior rendimento tendem a buscar mais consultas particulares quando há necessidade, porém, numa escala de consumo desses serviços ainda muito abaixo da realizada pelas mulheres.

Esses comportamentos diferenciados dificultam isolar os efeitos de sexo ou gênero na determinação de incidências de doenças entre homens ou mulheres, uma vez que um maior número de diagnósticos pode estar relacionado justamente ao fato de as mulheres buscarem mais os serviços de saúde por estarem mais atentas ao seu próprio estado de saúde. Em relação, por exemplo, às doenças crônicas não transmissíveis (DCNT), pela PNS, $56,9 \%$ das mulheres e $46,5 \%$ dos homens de 18 anos ou mais de idade referiram ter diagnóstico de ao menos uma doença crônica em 2019. A hipertensão arterial foi a DCNT mais referida, sendo diagnosticada em $26,4 \%$ das mulheres e $21,1 \%$ dos homens. Porém, foram observadas diferenças no aspecto preventivo e de acompanhamento da doença, com maior percentual de homens que nunca mediram pressão arterial ( $2,1 \%$ contra $1,2 \%)$, maior proporção de mulheres hipertensas com assistência médica no último ano $(74,5 \%$ contra $68,9 \%$ dos homens) e entre os que tomaram todos os medicamentos prescritos para controle da pressão nas duas últimas semanas $(89,6 \%$ contra $83,1 \%)$ (Tabela 2 ).

A segunda maior incidência de DCNT investigada pela pesquisa foi problema crônico na coluna, para a qual $24,5 \%$ das mulheres e $18,3 \%$ dos homens referiram ter diagnóstico e que, conforme apontado por Barata ${ }^{7}$, pode estar associado aos tipos distintos de trabalho remunerado e não remunerado desempenhados por homens $\mathrm{e}$ mulheres (especialmente os de caráter reprodutivo, monótono e desgastante por muitas horas realizado fundamentalmente pelas mulheres). DORT, atrite e reumatismo também apareceram com incidência bem maior entre as mulheres. $\mathrm{O}$ diagnóstico de depressão foi referido por 10,2\% da população adulta, porém com incidência quase três vezes superior para mulheres em relação aos homens $(14,1 \%$ e $5,1 \%$ respectivamente). Diabetes e colesterol alto também acometiam mais mulheres que homens, mas chama atenção o percentual de homens que nunca fizeram exame de sangue para medir glicemia em comparação às mulheres: $9,2 \%$ e $3,7 \%$ respectivamente (Tabela 2).

Todas as DCNT investigadas pela PNS apresentaram aumento de incidência em relação a 
Tabela 2. Proporção de homens e mulheres de 18 anos ou mais de idade que referiram diagnóstico de doenças crônicas não transmissíveis - Brasil, 2019.

\begin{tabular}{|c|c|c|c|c|}
\hline Doenças Crônicas Não-Transmissíveis & $\begin{array}{c}\text { Total } \\
(\%)\end{array}$ & $\begin{array}{c}\text { Homem } \\
(\%)\end{array}$ & $\begin{array}{c}\text { Mulher } \\
(\%)\end{array}$ & $\begin{array}{c}\text { Diferença } \\
\text { H-M } \\
\text { (pontos } \\
\text { percentuais) }\end{array}$ \\
\hline Depressão & 10,2 & 5,1 & 14,1 & 9,0 \\
\hline Artrite ou reumatismo & 7,6 & 3,7 & 11,0 & 7,3 \\
\hline Colesterol alto & 14,6 & 11,1 & 17,6 & 6,5 \\
\hline Problema crônico de coluna & 21,6 & 18,3 & 24,5 & 6,2 \\
\hline Hipertensão arterial & 23,9 & 21,1 & 26,4 & 5,3 \\
\hline Outra doença mental (1) & 6,5 & 4,1 & 8,6 & 4,5 \\
\hline Asma & 5,3 & 4,4 & 6,1 & 1,7 \\
\hline DORT (Distúrbio Osteomuscular Relacionado ao Trabalho) & 2,5 & 1,7 & 3,2 & 1,5 \\
\hline Diabetes & 7,7 & 6,9 & 8,4 & 1,5 \\
\hline Câncer & 2,6 & 2,1 & 2,9 & 0,8 \\
\hline Doença do coração & 5,3 & 4,9 & 5,6 & 0,7 \\
\hline Insuficiência renal crônica & 1,5 & 1,4 & 1,6 & 0,2 \\
\hline AVC & 2,0 & 2,0 & 2,0 & 0,0 \\
\hline
\end{tabular}

Nota: (1) Esquizofrenia, transtorno bipolar, psicose ou TOC.

Fonte: IBGE, Pesquisa Nacional de Saúde 2019.

2013 e, para boa parte delas, o atendimento era realizado pela Unidade Básica de Saúde. Por exemplo, relataram última consulta em uma UBS $46,6 \%$ dos hipertensos, 40,9\% dos diabéticos e $29,7 \%$ daqueles com diagnósticos de depressão. Isso representa uma pressão por recursos e ampliação dos serviços na entrada das pessoas no SUS, que, em função da maior incidência de DCNT nas mulheres, podem afetar proporcionalmente mais essas que os homens, conforme veremos de forma mais detida na próxima seção.

Por fim, no que se refere à obtenção dos remédios necessários à continuidade de tratamentos, $16,3 \%$ das pessoas conseguiram obter todos os medicamentos que foram receitados no serviço público de saúde e 15,0\% indicaram ter conseguido parte deles. Existe um pequeno diferencial por sexo, em que 32,3\% das mulheres conseguiram todos ou alguns dos medicamentos desta forma, contra 28,8\% dos homens. Padrão similar ocorre quando se verifica a diferença por cor ou raça, com pequenas diferenças nas quais as pessoas de cor ou raça preta ou parda conseguiram todos ou alguns dos medicamentos em maior proporção (33,8\% e 28,0\% para os brancos). Em 2013, a proporção de pessoas que conseguiram obter os medicamentos no serviço público era similar, com $15,6 \%$ obtendo todos e $18,5 \%$ obtendo parte dos medicamentos. Os diferenciais por sexo e cor ou raça são similares aos observados em 2019. Para 2008, os resultados não são diretamente comparáveis porque a sequência do questionário é outra, mas os resultados indicaram que $32,5 \%$ das pessoas afirmaram obter gratuitamente todos os medicamentos receitados, e $15,1 \%$ obteve parte deles desta forma.

\section{Fatores determinantes para o acesso e uso dos serviços de saúde no Brasil}

Pinheiro et al. ${ }^{14}$ defendem que apesar de seu caráter subjetivo, a autoavaliação do estado de saúde é frequentemente utilizada em inquéritos populacionais e tem especial relevância na explicação do uso de serviços de saúde. Conforme visto na seção anterior, mulheres e, em especial, pessoas de cor ou raça preta ou parda, foram os que menos referenciaram estados de saúde bom ou muito bom na PNS 2019. Ademais, essa autoavaliação da saúde vem piorando ao longo da última década para todos os grupos de sexo e cor ou raça com intensidades distintas. Entre 2008 e 2019, homens brancos reduziram sua avaliação do estado de saúde como bom ou muito bom em 2,9\%, enquanto mulheres brancas reduziram em $7,4 \%$, homens pretos ou pardos em 10\% e mulheres pretas ou pardas em 13\% (Gráfico 1). Para investigar se essa autopercepção diferenciada contribuiu para distintos acessos e usos dos serviços de saúde, os resultados apresentados nessa seção foram calculados considerando apenas os registros nos quais o informante foi a própria pessoa, excluindo assim os casos em que a resposta foi dada por outro morador ou por pessoa não mo- 


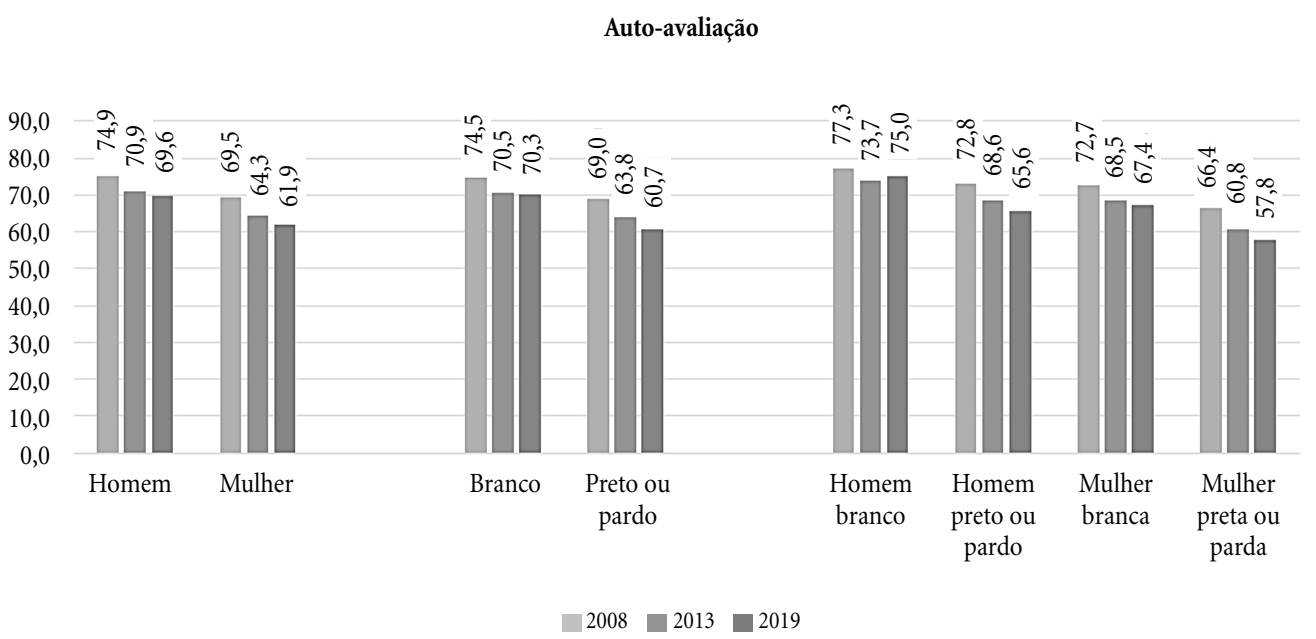

Gráfico 1. Proporção (\%) de homens e mulheres, por sexo e cor ou raça, que referenciaram estados de saúde como bom ou muito bom - Brasil, 2008/2013/2019.

Nota: Não são apresentados resultados para pessoas de cor ou raça amarela ou indígena.

Fonte: IBGE, Pesquisa Nacional de Saúde 2013/2019 e Pesquisa Nacional por Amostra de Domicílios 2008.

radora, além dos casos em que esta informação não estava disponível.

Conforme visto anteriormente, as séries históricas mostram que homens e mulheres tendem a buscar atendimento médico para tratamento da própria saúde de forma bastante diferenciada. As mulheres, mais que os homens, declararam ter consultado médico no último ano, porém essa diferença se reduziu ao longo da série. Em 2019, a diferença era de 12,7 pontos percentuais (p.p.) a favor das mulheres $(84,3 \%$ contra $71,6 \%)$; em 2013 de 15,4 p.p. e em 2008, 19,3 p.p. Mais uma vez, cor ou raça contribuiu para as diferenças encontradas, com mulheres brancas, em 2019, consultando médico no último ano em proporção maior que as pretas ou pardas $(86,4 \%$ e $82,8 \%)$ e situação similar encontrada entre homens brancos $(75,8 \%)$ e pretos ou pardos $(68,3 \%)$. O indicador teve aumento para todos os grupos analisados, mas foram os homens que proporcionalmente apresentaram maior crescimento na procura por consulta médica no último ano, $19,8 \%$ contra $6,5 \%$ das mulheres.

A piora geral na percepção da saúde pode estar associada a essa maior procura por atendimento médico uma vez que pessoas com autoavaliação mais positiva tendem a procurar menos o serviço de saúde. A proporção de pessoas que teve consulta médica em até um ano, entre aque- les que indicam ter saúde boa ou muito boa, foi 10,9 p.p. inferior aos demais em 2019, diferença menor que as observadas em 2013 (13,4 p.p.) e 2008 (17,7 p.p). Em 2019, entre os homens que avaliaram sua saúde como regular, ruim ou muito ruim, $80,2 \%$ se consultaram no último ano e, entre os que avaliaram seu estado de saúde como bom ou muito bom, $67,9 \%$ teve consulta no mesmo período (diferença de 12,3 p.p). Entre as mulheres, os percentuais foram, respectivamente, $81 \%$ e $89,6 \%$ (diferença de 8,6 p.p). A desagregação por sexo se mostrou mais determinante que a cor ou raça das pessoas. Mesmo entre o grupo que teve sua última consulta médica no período de até um ano, as mulheres consultaram médicos mais vezes, em média 4,5 consultas, contra 3,5 dos homens. Novamente, a autoavaliação cumpre um papel relevante, com o grupo de saúde boa ou muito boa apresentando em média duas consultas a menos no período de 12 meses do que os demais (Tabela 3).

A PNS também estimou que 39 milhões de pessoas $(18,6 \%$ da população) buscaram atendimento médico nas duas semanas que antecederam a entrevista. Ao considerar apenas as respostas em que a própria pessoa deu a informação sobre si mesma, o percentual passa para $22,7 \%$ da população. Diferenciais de gênero e a autoavaliação de saúde mantiveram seu papel explicativo. 
Tabela 3. Proporção de homens e mulheres, por sexo e cor ou raça, que realizaram consulta no último ano e média de consultas no último ano, segundo autoavaliação do estado de saúde - Brasil, 2008/2013/2019.

\begin{tabular}{|c|c|c|c|c|c|}
\hline Sexo e/ou cor ou raça & $\begin{array}{c}2008 \\
(\%)\end{array}$ & $\begin{array}{c}2013 \\
(\%)\end{array}$ & $\begin{array}{c}2019 \\
(\%)\end{array}$ & $\begin{array}{c}\text { Variação } \\
\text { percentual } \\
2008 / 2019(\%)\end{array}$ & $\begin{array}{c}\text { Média de } \\
\text { consultas } \\
\text { em } 2019\end{array}$ \\
\hline \multicolumn{6}{|l|}{ Com consulta último ano } \\
\hline Homem & 59,8 & 64,5 & 71,6 & 19,8 & 3,5 \\
\hline Mulher & 79,1 & 79,9 & 84,3 & 6,5 & 4,5 \\
\hline Branco & 74,1 & 77,2 & 82,3 & 11,0 & 4,1 \\
\hline Preto ou Pardo & 69,1 & 71,3 & 77,3 & 11,9 & 4,2 \\
\hline Homem branco & 62,8 & 68,7 & 75,8 & 20,8 & 3,6 \\
\hline Homem preto ou pardo & 57,1 & 60,9 & 68,3 & 19,7 & 3,4 \\
\hline Mulher branca & 81,3 & 82,5 & 86,4 & 6,3 & 4,4 \\
\hline Mulher preto ou pardo & 77,1 & 77,7 & 82,8 & 7,4 & 4,6 \\
\hline \multicolumn{6}{|c|}{ Pessoas com consulta último ano e auto-avaliação boa ou muito boa da saúde } \\
\hline Homem & 54,0 & 60,2 & 67,9 & 25,7 & 2,8 \\
\hline Mulher & 75,3 & 76,1 & 81,0 & 7,7 & 3,8 \\
\hline Branco & 69,9 & 73,3 & 79,4 & 13,7 & 3,5 \\
\hline Preto ou Pardo & 63,2 & 65,9 & 72,3 & 14,5 & 3,3 \\
\hline Homem branco & 57,5 & 64,8 & 72,9 & 26,7 & 3,0 \\
\hline Homem preto ou pardo & 50,6 & 55,8 & 63,3 & 25,2 & 2,7 \\
\hline Mulher branca & 78,1 & 79,2 & 84,0 & 7,6 & 3,8 \\
\hline Mulher preto ou pardo & 72,3 & 73,0 & 78,5 & 8,5 & 3,7 \\
\hline \multicolumn{6}{|c|}{ Pessoas com consulta último ano e auto-avaliação regular, ruim ou muito ruim da saúde } \\
\hline Homem & 77,1 & 75,2 & 80,2 & 4,0 & 4,9 \\
\hline Mulher & 88,0 & 86,9 & 89,6 & 1,9 & 5,6 \\
\hline Branco & 86,6 & 86,4 & 89,1 & 2,9 & 5,5 \\
\hline Preto ou Pardo & 82,5 & 80,7 & 85,1 & 3,2 & 5,3 \\
\hline Homem branco & 80,6 & 79,7 & 84,5 & 4,9 & 5,4 \\
\hline Homem preto ou pardo & 74,6 & 72,0 & 77,9 & 4,4 & 4,5 \\
\hline Mulher branca & 89,8 & 89,9 & 91,3 & 1,7 & 5,6 \\
\hline Mulher preto ou pardo & 86,7 & 85,0 & 88,7 & 2,4 & 5,6 \\
\hline
\end{tabular}

Nota: Não são apresentados resultados para pessoas de cor ou raça amarela ou indígena.

Fonte: IBGE, Pesquisa Nacional de Saúde 2013/2019 e Pesquisa Nacional por Amostra de Domicílios 2008.

Este último fator foi responsável por uma diferença média de 14,2 p.p. na proporção de busca por atendimento no período, diferença que se manteve relativamente estável para as desagregações de sexo e cor ou raça. Novamente, o grupo com maior procura de atendimento médico foi o das mulheres brancas. Entre as mulheres brancas com autoavaliação menos positiva da própria saúde, o percentual de busca por atendimento médico nas duas semanas anteriores foi de $36,8 \%$. Por outro lado, os homens pretos ou pardos com autoavaliação positiva representaram o grupo com menor busca (12,0\% procuraram atendimento).

Entre as mulheres que procuraram atendimento nas duas semanas anteriores, $3,6 \%$ o fizeram por razões relacionadas à gravidez. Desconsiderando este grupo, observa-se que o principal motivo de busca por atendimento, tanto para homens quanto para mulheres, foi doença (dor, febre, diarreia etc.) ou continuação de tratamento de doença ( $49,2 \%$ para mulheres e $45,8 \%$ para homens). Por sua vez, homens buscaram mais atendimento de saúde relacionado a acidente, lesão ou fratura, principalmente os de cor ou raça preta ou parda, chegando a $8,7 \%$ dos motivos para este grupo (6,4\% para homens brancos).

O local de procura do primeiro atendimento não apresentou diferença significativa por sexo, com aproximadamente 59\% buscando atendimentos em hospitais ou ambulatórios públicos e $38 \%$ em privados. Contudo, existe diferença por cor ou raça, em que os pretos ou pardos buscaram mais atendimento público $(68,0 \%$ contra $29,4 \%$ privados) em comparação com os brancos 
(48,7\% contra $48,9 \%$ privados). No geral, $71,1 \%$ das pessoas conseguiram atendimento na primeira tentativa, com alguns diferenciais relacionados principalmente ao motivo da procura. Este número representa uma queda considerável em comparação ao observado em investigações anteriores, quando a proporção de pessoas que conseguiram obter atendimento médico na primeira tentativa foi de $95,6 \%$ em 2008 e 94,6\% em 2013. Contudo, parte desta diferença pode ser associada à atualização do quesito, implementada na edição 2019 da PNS, na qual foi inserida a categoria de resposta "Foi agendado para outro dia/outro local" para a pergunta sobre atendimento na primeira procura, que contemplou $26,0 \%$ das pessoas.

Para melhor quantificar os fatores que influenciaram no sucesso na primeira busca por atendimento, foi ajustado um modelo de regressão logística, considerando como variável resposta o sucesso nesta primeira busca, e como variáveis explicativas sexo, cor ou raça, grupos de idade, autoavaliação do estado de saúde, motivo da procura por atendimento e local da procura, controlando por Unidades da Federação. Para o ajuste do modelo também foram considerados apenas os casos em que a própria pessoa deu a informação sobre si mesma, totalizando 25.150 registros na amostra da PNS 2019 de pessoas que informaram ter buscado atendimento nas duas semanas anteriores.

Inicialmente, foi ajustado o modelo completo, considerando todas as variáveis, inclusive um termo de interação entre sexo e cor ou raça. A inclusão deste termo de interação não trouxe ganho estatisticamente significativo ao modelo pelo teste de Wald ( $\mathrm{p}=0,595)$. Os demais termos foram mantidos no modelo final. A Tabela 4 ilustra os resultados obtidos, juntamente com as razões de chances (RC) e intervalos de $95 \%$ de confiança. O teste de Archer-Lemeshow indicou bom ajuste do modelo aos dados, com p-valor 0,573. A inclusão dos termos referentes às Unidades da Federação apresentou ganho significativo ao modelo, pelo teste de Wald ( $\mathrm{p}<0,001)$. Contudo, por não se tratar de um grupo de interesse analítico neste trabalho, e pela quantidade de coeficientes (26), estes não foram apresentados na referida tabela.

Sexo, idade, motivo e local da procura foram os fatores que mais se associaram a conseguir atendimento na primeira busca, de forma que mulheres tinham menos chance de conseguir realizar a consulta na primeira tentativa $(\mathrm{RC}=$ 0,86). Como apontado anteriormente, mulheres buscam atendimento mais frequentemente, enquanto homens tendem a fazê-lo quando sentem dores ou há algum problema mais grave de saúde, o que pode justificar que eles tenham mais chance de sucesso na primeira tentativa de atendimento. A diferença entre brancos e pretos ou pardos não foi estatisticamente significativa. Estudos anteriores indicam maior dependência do sistema público de saúde para pretos ou pardos, além de pior autoavaliação de saúde para estes grupos. Com a inclusão destes fatores no modelo logístico, a cor ou raça não apresentou diferencial estatisticamente significativo.

Existe diferença significativa para grupos de idade, com as idades mais elevadas apresentando menor chance de conseguir atendimento na primeira tentativa. Por sua vez, o motivo que se destacou com a maior chance de sucesso no atendimento foi acidente, já que os demais possuem $\mathrm{RC}$ abaixo de 1 . Também possuem maior chance de conseguir atendimento na primeira tentativa aqueles que o buscaram em locais privados.

Entre aqueles que não conseguiram atendimento na primeira tentativa, pouco mais da metade $(53,0 \%)$ tornou a buscá-lo, e, destes, aproximadamente nove em cada dez conseguiram ter seu atendimento de saúde realizado. De forma mais ampla, a proporção de pessoas que obteve sucesso na busca por seu atendimento de saúde, seja na primeira tentativa ou em alguma tentativa posterior, foi de aproximadamente $85 \%$.

\section{Conclusões}

O cenário traçado pela PNS 2019 ganhou novos contornos no atípico ano de 2020. Informações como a percepção do próprio estado de saúde, existência prévia de doenças crônicas, acesso a medicamentos e a utilização dos serviços de atenção primária à saúde são importantes para delinear o perfil dos atendimentos médicos e dos usuários e usuárias do sistema de saúde, ao mesmo tempo que permitem avaliar demandas emergenciais latentes e a capacidade de atendimento e resolutividade do sistema, justamente os aspectos de atenção à saúde mais pressionados em 2020 pela pandemia da COVID-19.

A análise dos dados mostrou a perene importância do SUS para a realização do direito constitucional à saúde da população brasileira, não obstante as diversas questões estruturais que limitam hoje sua atuação, em especial, aquelas concernentes ao seu financiamento que impactam profundamente a oferta e a qualidade dos serviços oferecidos. Não obstante, três quartos da população brasileira não possui acesso à saúde via planos privados e dependem exclusivamen- 
Tabela 4. Coeficientes do modelo logístico para sucesso na primeira busca por atendimento e respectivas razões de chances.

\begin{tabular}{lcc}
\hline \multicolumn{1}{c}{ Fator } & Coeficiente & $\begin{array}{c}\text { Razão de chances } \\
\text { (IC 95\%) }\end{array}$ \\
\hline Intercepto & 1,97 & - \\
Sexo (ref: homem) & - & - \\
Mulher & $-0,15$ & $0,86(0,79 ; 0,95)$ \\
Cor ou raça (ref: branca) & - & - \\
Preta ou parda & 0,07 & $1,07(0,96 ; 1,19)$ \\
Grupos de idade (ref: menos de 30 anos) & - & - \\
30 a 49 anos & $-0,16$ & $0,85(0,74 ; 0,98)$ \\
50 a 64 anos & $-0,25$ & $0,78(0,67 ; 0,90)$ \\
65 anos ou mais & $-0,32$ & $0,73(0,62 ; 0,85)$ \\
Autoavaliação de saúde (ref: muito ruim, ruim ou regular) & - & - \\
Bom ou muito bom & $-0,07$ & $0,93(0,84 ; 1,04)$ \\
Motivo da procura (ref: Acidente) & - & - \\
Doença & $-0,65$ & $0,52(0,40 ; 0,68)$ \\
Exames & $-1,34$ & $0,26(0,20 ; 0,35)$ \\
Prevenção ou acompanhamento & $-1,32$ & $0,27(0,20 ; 0,35)$ \\
Local da procura & & - \\
(ref: hospitais ou ambulatórios públicos) & - & $1,17(1,04 ; 1,31)$ \\
Hospitais, consultórios ou ambulatórios privados & 0,15 &
\end{tabular}

Fonte: IBGE, Pesquisa Nacional de Saúde 2019.

te do serviço público de saúde. As crises social, econômica e política que marcaram o período de análise dos dados, em especial os anos recentes, associadas ao processo de envelhecimento populacional, mostram uma piora na percepção das pessoas sobre o seu estado geral de saúde, maior incidência de DCNT e um aumento na procura por atendimentos médicos.

As desigualdades de gênero e raciais, enquanto estruturas fundantes da sociedade brasileira, se refletem nos dados ora analisados. A PNS 2019 confirmou a tendência de mulheres serem mais cuidadosas com a saúde que homens. Não devemos, no entanto, avaliar mulheres e homens como grupos homogêneos, uma vez que brancos e pretos/pardos apresentam comportamentos e acessos diferenciados no que tange ao cuidado com a saúde. $\mathrm{O}$ fato de homens terem maiores chances de conseguir atendimento na primeira tentativa relaciona-se ao fato de que boa parte desses primeiros atendimentos se referiram a causas externas, que exigem atendimento imediato de urgência e é causa característica do comportamento masculino.

Nesse sentido, os resultados apontam que o estudo dos papéis de gênero na sociedade, exponenciados pela cor ou raça de homens e mulheres, são fundamentais para o entendimento dos fatores determinantes do acesso e utilização dos serviços de saúde. São esses comportamentos socialmente esperados que homens e mulheres desempenhem em função do seu sexo biológico que ainda hoje permeiam a maior exposição de homens a situações de risco e a dedicação desigual de mulheres aos afazeres e cuidados de outras pessoas. Tais comportamentos impactam o uso e o acesso aos serviços de saúde, seja em função das mulheres alcançarem idades mais avançadas em maior proporção que os homens, seja pela maior atenção crítica que elas conferem à sua saúde $\mathrm{e}$ daqueles de quem cuidam, seja pelos efeitos das atividades laborais e reprodutivas desempenhadas por cada sexo, seja pelo impacto emocional advindo de todo esse conjunto de fatores que atingem diferenciadamente homens e mulheres.

Todavia, uma vez vencidas as dificuldades e fatores que levam cada um a acessar o sistema de saúde, a universalidade e integralidade preconizadas no SUS tendem a reduzir iniquidades e impactam menos nas chances de prosseguir no atendimento ou na obtenção de medicamentos, internações e tratamentos. Disso deduz-se que investir na garantia do atendimento resolutivo e eficiente em todos os níveis de saúde, notadamente na porta de entrada de um sistema tão complexo como o SUS, pode não solucionar 
questões profundas da conformação da sociedade brasileira, como as desigualdades de gênero, mas se mostram fundamentais na equalização de oportunidades, realização de direitos, promoção de bem-estar e qualidade de vida.

\section{Colaboradores}

B Cobo trabalhou na organização do projeto, concepção, revisão bibliográfica, análise dos dados, redação e revisão final. C Cruz trabalhou na concepção, revisão bibliográfica, redação e revisão final. PC Dick trabalhou na concepção, metodologia, análise dos dados, redação e revisão final. 


\section{Referências}

1. Instituto Brasileiro de Geografia e Estatística (IBGE). Pesquisa nacional de saúde: 2019: informações sobre domicílios, acesso e utilização dos serviços de saúde: Brasil, grandes regiões e unidades da federação. Coordenação de Trabalho e Rendimento. Rio de Janeiro: IBGE; 2020. 85p. [acessado 2021 jan 3]. Disponível em: https://biblioteca.ibge.gov.br/index.php/biblioteca-catalogo?view $=$ detalhes \&id $=2101748$

2. Instituto Brasileiro de Geografia e Estatística (IBGE). Pesquisa nacional de saúde: 2013: acesso e utilização dos serviços de saúde, acidentes e violências: Brasil, grandes regiões e unidades da federação. Coordenação de Trabalho e Rendimento. Rio de Janeiro: IBGE; 2015. 106 p. [acessado 2021 jan 3]. Disponível em: http://portalarquivos.saude.gov.br/images/pdf/2015/junho/09/ PNS-2013-1.pdf

3. Instituto Brasileiro de Geografia e Estatística (IBGE). Pesquisa Nacional por Amostra de Domicílios: Brasil, grandes regiões e unidades da federação. Coordenação de Trabalho e Rendimento. Rio de Janeiro: IBGE; 2008. 126 p. (CD-ROM) [impresso]. [acessado 2021 jan 3]. Disponível em: https://static.scielo.org/scielobooks/48z26/pdf/barata-9788575413913.pdf

4. R Foundation for Statistical Computing. R: A language and environment for statistical computing. Version 4.0.0. Vienna, Austria: 2020.

5. Lumley T. Complex Surveys: A Guide to Analysis Using R. EUA: John Wiley and Sons; 2010.

6. Archer KJ, Lemeshow S. Goodness-of-fit test for a logistic regression model fitted using survey sample data. Stata J 2006; 6(1):97-105.

7. Brasil. Ministério da Saúde (MS). Secretaria de Atenção Primária à Saúde (SAPS) O que é Atenção Primária? [acessado 2021 jan 3]. Disponível em: https://aps. saude.gov.br/smp/smpoquee

8. Krieger N. Genders, sexes, and health: What are the connections - and why does it matter? Int J Epidemiol 2003; [cited 2021 Jan 3]. Available from: https://pdfs.semanticscholar.org/d174/3ebd1a10afe6398bb795c5f83cc704094f18.pdf?_ga=2.176119914. 912630888.1609179086-66620828.1609179086

9. Gahagan J, Gray K, Whynach A. Sex and gender matter in health research: addressing health inequities in health research reporting. Int J Equity Health 2015 p. 14:12. [cited 2021 Jan 3]. Available from: https:// www.researchgate.net/publication/271647755_Sex_ and_gender_matter_in_health_research_Addressing_health_inequities_in_health_research_reporting/link/55258bfe0cf24b822b405516/download
10. Barata RB. Relações de gênero e saúde: desigualdade ou discriminação? In: Como e por que as desigualdades sociais fazem mal à saúde [online]. Rio de Janeiro: Editora Fiocruz; 2009. Temas em Saúde collection, p. 73-94. [acessado 2021 jan 3]. Disponível em: SciELO Books <http://books.scielo.org $>$.

11. Cobo B. O trabalho doméstico no pós-pandemia. Terapia Política. 2020. [acessado 2021 jan 3]. Disponível em: https://terapiapolitica.com.br/2020/06/25/o-trabalho-domestico-no-pos-pandemia/

12. Gomes R, Nascimento EF, Araujo FC. Por que os homens buscam menos os serviços de saúde do que as mulheres? As explicações de homens com baixa escolaridade e homens com ensino superior. Cad Saude Publica 2007; 23(3):565-574.

13. Levorato CD, Mello LM, Silva AS, Nunes AA. Fatores associados à procura por serviços de saúde numa perspectiva relacional de gênero. Cien Saude Colet 2014; 19(4):1.263-1.274.

14. Pinheiro RS, Viacava F, Travassos C, Brito AS. Gênero, morbidade, acesso e utilização de serviços de saúde no Brasil. Cien Saude Colet 2002; 7(4):687-707.

Artigo apresentado em 05/01/2021

Aprovado em 30/04/2021

Versão final apresentada em 02/05/2021

Editores-chefes: Maria Cecília de Souza Minayo, Romeu Gomes, Antônio Augusto Moura da Silva 